\title{
DE COMUNIDADES INVENTADAS A COMUNIDADES INVISIBLES: HACIA UN MARCO TEÓRICO PARA EL ESTUDIO DE LOS YUMANOS DE BAJA CALIFORNIA ${ }^{1}$
}

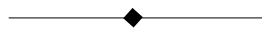

\begin{abstract}
RESU MEN
Este articulo es una aproximación al proceso de construcción de redes sociales y etnicidad entre los indígenas yumanos de Baja California, México. A partir de su cuestionamiento a los enfoques tradicionales que reiteradamentehan anunciado la extinción y asimilación de estos grupos, estearticulo sugiere un nuevo marco teórico para el estudio de la supuesta disipación de estas poblaciones étnicamente diferenciadas. Conceptos central es de esta propuesta teórica son los conceptos decomunidad transnacional, hiper espacio y polibio, los cuales según el autor, aplicados al trabajo de campo, pueden conducirnos a revelar las formas en que los yumanos permanecen comprometidos en la elaboración de nuevas formas de organización social y auto-referencia étnica, a través de la construcción de amplias redes sociales queincluyen (aunqueno selimitan) a los grupos lingüísticamenterelacionados que habitan en los Estados Unidos.
\end{abstract}

\section{A BSTRACT}

This paper focuses on the construction of social networks and ethnicity among the Yuman indigenous peopleof Baja California, M exico. By questioning thetraditional view of the Yumans as decimated and culturally assimilated groups, this paper suggests a new theoretical framework to approach the alleged dissipation of distinctive Yuman populations. Central concepts of this framework arethenotions of transnational community, hyperspace and polybian. According to the author, these concepts applied to field-research may reveal how the Yumans remain engaged in the elaboration of new forms of social organization and ethnic self-references, through the construction of wide social networks that include (although it is not limited to) the linguistically-related groups living in the United States.

\footnotetext{
* Investigador del instituto de InVestigaciones Históricas de la uabc, CANDIDATo a dortor en antropología en la Universidad Estatal de Arizona. Correo electrónico: everardo@faro.ens.uabc.mx

${ }^{1}$ Investigación en proceso. Proyecto financiado por el Fideicomiso para la Cultura México-EuA (Fidemexusa).
} 


\section{INTRODUCCIÓN}

Tradicional mente, los grupos indígenas que viven en el área geográfica que ha sido subsecuentementellamada la Gran Chichimeca, Á rido A mérica, o la frontera México-Estados Unidos, han sido tratados de manera marginal por la antropología. De ser considerados sólo una serie de bandas salvajes, y técnicamente una serie de linajes nómadas de cazadores y recolectores, estos grupos arribaron al siglo XXI bajo dos consideraciones principales: 1) que se encuentran experimentando un dramático proceso deextinción, y/ o 2) un inevitableproceso de asimilación cultural.

Partiendo de estas premisas, los indígenas de la frontera han sido losgrandes ignorados por los estudiosos delos fenómenos relacionados con la presenteera detransnacional ismo. Al final delos años setentas, los especialistas sobre frontera y migración empezaron a atender las experiencias migratorias de grupos procedentes de Meso América, África, o los países caribeños, ignorando a los grupos indígenas que habían establecido su residencia durante varios siglos en las inmediaciones delo quehoy es la frontera M éxico-Estados Unidos. A sí, los argumentos sobre transnacionalismo que empezaron a generarse a partir de la existencia de múltiples procesos que trascienden las fronteras geográficas, políticas, y culturales, fueron elaborados con el estudio deexperiencias migratorias demixtecos, zapotecos, purépechas, maya-quiché, grupos no indígenas de Haití, Puerto Rico, Cuba, Granada, etcétera, pero no de los grupos que iniciaron de manera temprana la construcción de campos sociales sobre dichas fronteras: los cucapá, los yaqui, los tohono o'odham, los hia-ced o'odham, los kikapú, y particularmente, los yumanos deBaja California.

De esta manera, sobre la base de un reducido número de estudios de caso, la antropología transnacional ha arribado a tres generalizaciones centrales: 1) que el incremento de una migración multidireccional ha venido a cuestionar la noción tradicional de comunidad geográficamentedel imitada y autocontenida (Basch, et al. 1994; Rouse, 1991); 2) que esta nueva migración multidireccional sin embargo, gravita al rededor de áreas geográficas con una elevada demanda de fuerza de trabajo, conocidas como ciudades globales (Sassen, 1998; 
Chambers, 1994); 3) que en estas ciudades globales los inmigrantes tienden a recrear aspectos distintivos desu cultura, tal y como prácticas cooperativas y solidarias, a través de procesos de reterritorialización (Kearney, 1995) o barrialización (Vélez, 1996), que resultan en la creación de comunidades vástago (Chávez, 1990), nuevos escenarios étnicos (A ppadurai, 1991) o locis culturales (Rouse, 1992).

Aunquelas anteriores conclusiones pueden ser válidas para ciertos casos, y más concretamente para los casos hasta entonces estudiados, este artículo sostiene que el reanál isis de los grupos indígenas de la frontera desde el punto de vista de las nuevas perspectivas antropológicas, puede arrojar hallazgos interesantes. Tal y como lo afirma Michael Kearney (1995), considerando las nuevas elaboraciones sobre redes sociales, etnicidad y comunidad, los antropólogos pueden reevaluar los tradicional mente llamados procesos de extinción indígena y asimilación cultural. Más aún, en mi opinión, al reorientar nuestro enfoque sobre los indígenas de la frontera, podremos reconocer, también, diferentestipos deredessocialesy patronesmigratorios, deaquellos regularmente discutidos en la moderna literatura antropológica.

Partiendo deesta consideración, y haciendo uso delas nociones de Michael Kearney (1996) sobrehiperespacio y polibio, esteartículo sugiere un nuevo marco teórico para el estudio de los procesos observados entre los indígenas yumanos de Baja Cal ifornia en la última parte de este siglo. A través de la evaluación de las redes sociales y etnicidad de los yumanos, este artículo no sólo cuestiona las tradicionales nociones de extinción indígena y asimilación cultural, como procesos supuestamente experimentados por estos grupos indígenas, sino también, la supuesta tendencia de todo migrantea migrar hacia centros de contratación con una elevada demanda de fuerza de trabajo, y constituir así comunidades vástago perfectamenteidentificables. Otros aspectos que este artículo sostiene son: 1) que las comunidades yumanas, usualmente concebidas con una serie de características románticas, son invenciones ilusorias del Estado y los antropólogos clásicos; 2) quelos yumanos deBaja Cal ifornia han resistido históricamente a esta forma tradicional de comunidad, a través del boicoteo a los proyectos indigenistas de orientación comunitaria, a través de la deconstrucción de estas comunidades inventadas como sitios de 
residencia de las cabezas de linaje, y a través de la construcción de comunidades invisibles, elaboradas sobre la base de su propia organización social.

\section{ANTECEDENTES}

La familia etnolingüística yumana comprende aproximadamente quince grupos étnicos, distribuidos a lo largo de los desiertos de Baja California y Sonora, en M éxico, y A rizona y California en los Estados Unidos. En Baja California, cinco de estos grupos étnicos han sido tradicionalmenteasociados a ocho comunidades sedentarias conocidas como ejidos, las cuales se encuentran dispersas dentro de un área geográfica localizada entrelos 30 grados latitud nortey la línea fronteriza M éxico-Estados Unidos. Estos grupos y sus correspondientes comunidades son, los cucapá del Ejido El M ayor Indígena, situado en las márgenes del Río Colorado; Ios kiliwa del Ejido Arroyo de León en el Valle de la Trinidad, entre la Sierra de Juárez y la sierra de San Pedro Mártir; Los pai-pai delos ejidos Santa Catarina y San Isidoro, al suroeste de la Sierra de Juárez; los t'pai de los ejidos La Huerta y San A ntonio Necua, al noroestedela Sierra de Juárez; y los k'miai delos ejidos San José de la Zorra y Juntas de N ejí, al norte de la Sierra de Juárez.

Como lo habrá notado todo aquel que haya visitado al guna deestas comunidades, una de sus principales características es la desolación y la notablepresencia de una población predominantementedeancianos. El caso más extremo es seguramente el del ejido kiliwas, conocido también como Arroyo de León. En esta comunidad, lo primero que llama la atención es una iglesia blanca rodeada por cuatro pequeñas casas en medio de un desolado parajelleno de piedras, entre las cuales se pierden distantes caseríos separados incluso por kilómetros, y una población visible de no más de 15 personas. Y es que en efecto, los asentamientos yumanos más poblados son los de Santa Catarina y La Huerta con apenas 169 y 142 habitantes respectivamente, seguidos por San Isidoro y San José de la Zorra, con 77 habitantes cada uno, y por último A rroyo de León con 33 indígenas. Deesta manera, en total, la población quehabita en éstos y el resto delos ocho ejidos indígenas, se compone de al rededor de 700 personas (Garduño, 1995). 
Como resultado de estos signos de desolación proyectados por las comunidades yumanas, historiadores, sociólogos, antropólogos, e incluso biólogos, han abonado la idea de extinción indígena a través de distintas hipótesis. Para algunos, la extinción de los yumanos sólo representala última partedeun proceso continuo queha tenido lugar de los siglos XVII al XX, y de sur al norte de Baja California, einiciada como resultado de la introducción de enfermedades epidémicas por los europeos (viruela, sarampión, sífilis, tuberculosis y tifoidea) (Aschmann, 1959; Martínez, 1991; León-Portilla, 1983; Dieguet, 1978). Parte de esta conclusión descansa generalmente sobre la siguiente información histórica. En 1772, se dice que el misionero jesuita Juan Jacobo Baegert (1942) estimó quela población indígenaal momento de arribar los colonizadores españoles, ascendía a 50,000 individuos que habitaban desdeel Cabo San Lucas al Río Colorado. Esta población se encontraba compuesta por 17gruposétnicos diferentes y cuatro familias etnolingüísticas: pericú, guaycura, yumano-peninsular (o cochimí) y Iosyumanos del norte(León-Portilla, 1983). En el siglo XIX, sin embargo, se dice quelossacerdotes dominicos no encontraron ningún pericú ni guaycura viviendo en su territorio tradicional localizado hacia el sur dela península de Baja California, sino solamente cerca de 10,000 cochimís y yumanos viviendo hacia el norte(Massey, 1949; Meigs, 1970; Cook, 1937). Más aún, hacia el final del periodo misional, el general Luis E. Torres, jefe político y militar en el Distrito Norte de Baja Cal ifornia registró una población de4424hablantes delenguayumana, viviendo en las sierras de Juárez y San Pedro Mártir, y en las inmediaciones del Río Colorado (Martínez, 1985). Finalmente, del primer tercio del presente siglo a la actualidad, geógrafos, etnógrafos e instituciones oficiales, han sugerido que menos de 1000 indígenas, pertenecientes solamentea una familia etnolingüísticay distribuidos entrecinco grupos étnicos, han sobrevivido al proceso decolonización, habitando exclusivamente en la parte norte de la península de Baja California (Meigs, 1939; INAH, 1972, 1978; INI, 1982, 1991).

Otros autores, por su parte, han sugerido otro tipo de hipótesis ciertamente creativas, que ubican las causas de dicha extinción hacia el siglo xx. Por ejemplo, destacando la el evada presencia de ancianos dentro delas comunidades indígenas, ha sido sugerido quela supuesta 
extinción indígena obedece a factores opuestos al de mortalidad. Algunos han planteado, por ejemplo, quela al teración en el estilo devida tradicional de estos grupos ha producido un cambio dramático en el consumo de un agave que abunda en la región y que se conoce con el nombre de palmilla, el cual posee cualidades anticonceptivas (Zárate y Cortez, comunicación personal). Otros han argumentado que debido al despojo territorial, estos grupos han experimentado una pérdida desu conciencia deprocreación y han empezado a practicar una especie de "etno-suicidio". De acuerdo con esta hipótesis, la presencia de grupos indígenas empezó a declinar cuando adquirieron conciencia de que habían perdido el bien heredable más valioso que tenían: su territorio tradicional (Ochoa, 1979). Una tercer y más sofisticada explicación que involucra una variedad de factores, es la expuesta por Miguel León-Portilla. Este historiador argumenta quelos misioneros, al concentrar a aquel las bandas semi nómadas en "cóngregas" o centros misionales, alteraron su milenaria adaptación al medio ambiente natural, y que una vez que este equilibrio fue al terado, estos indígenas empezaron a experimentar una serie de traumas cultural es psicológicosy hasta biológicos. El resultado deesteproceso, según León-PortiIla, fuela absorción del grupo por la vía del mestizaje, o más frecuentemente a través de la muerte de sus miembros (León-Portilla, 1985). Finalmente, Hinton y Owen (1957) han planteado que estos indígenas, mas que un proceso de extinción, empezaron a experimentar un proceso de proletarización a principios del siglo veinte, con su incorporación al desarrollo capitalista regional como vaqueros, mineros y jornaleros, y como consecuencia, esta proletarización condujo a la detribal ización-asimilación delosyumanos, al ser subsumidos bajo la cultura del vaquero.

No obstante lo sugerente de todas estas hipótesis, su exactitud es cuestionable. Primero, la idea de un proceso de extinción continuo iniciado en la época misional, no puede aplicarse al fenómeno observado entre la población indígena del norte de Baja California, porque nada nos dice acerca de lo ocurrido en el siglo xx con aquellos quesobrevivieron alas enfermedades epidémicas. Más aún, tal y como lo comprobó Roger Owen en los años cincuenta, la construcción de accesos y caminos rurales hizo posiblela introducción dela medicina 
moderna a las áreas indígenas, y como consecuencia, la elevada mortandad resultado de las enfermedades epidémicas disminuyó importantementeentreestas poblaciones. Dehecho Roger Owen (1959) encontró en 1958, que las condiciones sanitarias de los alimentos y el agua dentro del territorio indígena eran excelentes, por lo queel cuadro demorbi-mortalidad era similar al observado en lasáreas no indígenas. Con respecto a la hipótesis sobre la palmilla, podemos decir que no existeevidencia científica que confirmelas cualidades anticonceptivas deesta planta, y quela mayoría demis informantes han desacreditado esta teoría al afirmar que, pese a que sus abuel os y padres fueron asiduos consumidores de este agave, sus familias estuvieron compuestas por numerosos descendientes. Por otra parte, la teoría sobre etnosuicidio cayó por sí sola cuando extensas áreas de tierra fueron reconocidas legal mentecomo territorio indígena, y aún así, el supuesto descenso demográfico indígena continuó: como se hizo mención anteriormente, la población indígena en esta área cayó de 4,000 a 1,000 individuos a la vuelta del siglo XIX y de 1,000 a 700 personas en el siglo $x x$. Por último, aun cuando estoy de acuerdo en el hecho de que estas comunidades indígenas iniciaron un proceso de proletarización a principios del siglo Xx, cuestionaría el que este proceso esté conduciendo a una asimilación cultural generalizada. Como será discutido en la siguiente sección, el indicador observado por los antropólogos transcultural es como signo deextinción y asimilación, es simplementeel desvanecimiento deentidades quenunca han existido realmente. En mi opinión, las comunidades yumanas nunca han sido unidades sedentarias, cooperativas ni agrícolas como se ha creído, sino que su concepción con estas características, obedece a una invención ideológica del Estado y los antropólogos, que ha sido históricamente boicoteada por los mismos indígenas.

\section{LASCOMUNIDADESINVENTADASY SU DECONSTRUCCIÓN}

Los teóricos del transnacionalismo han señalado críticamente, que la antropología ha trabajado tradicionalmentecon la noción decomunidad entendida como una entidad espacial y culturalmente delimitada (Varese, 1994; Chávez, 1992; Rouse, 1991; Kearney, 1991). Deacuerdo 
con estos autores, las bases de dicha noción fueron elaboradas en principio por la antropol ogía colonial, la cual estableció como característica inherentea toda comunidad su local ización geográfica claramentediferenciada. Posteriormente, en los años sesenta, sobre esta noción fue desarrollado el concepto de comunidades corporadasy autocontenidas, portadoras de una cultura eidentidad étnica específica, y con una serie de características entre las cuales destaca la igualdad social y la solidaridad. Ejemplos deesta noción pueden encontrarse, primero, en las elaboraciones de Eric Wolf (1957), en las que se afirma que "Ia cohesión social comunitaria" es característica central deun "orden social tradicional"; un segundo ejemplo puede ser encontrado en la afirmación deSeton-Watson (1997), en el sentido de queademás de su delimitación geográfica, la comunidad se caracteriza por su sentido de solidaridad, la existencia de una cultura común y una conciencia comunal entresus miembros.

En la actualidad, sin embargo, esta noción de comunidad ha sido cuestionada por los especial istas. Por una parte, atendiendo la creciente movilidad transnacional y multidereccional que caracteriza a la era post-colonial, estos teóricos han revelado la existencia de poblaciones extremadamente dispersas que a pesar de esto, continúan reproduciendo sus lazos sociales por encima incluso de las fronteras políticas (Basch, et al., 1994; Rouse, 1991). Por otra parte, estos teóricos han empezado a cuestionar también, las esencialistas descripciones antropológicas de comunidades indígenas carentes de procesos de diferenciación interna y enmarcadas en una lógica social de romántica reciprocidad. Stefano Varese (1994) y Frank Cancian (1992) señalan, por ejemplo, que el sistema de cargos y el Ilamado gasto cívico-ceremonial, tradicional mente concebidos como mecanismos que impiden la formación declases sociales y grupos al interior delas fronteras comunitarias, necesitan ser reanalizados a la luz del nuevo contexto social global, del cual estas comunidades indígenas son inevitablementeparte. De acuerdo con Cancian, la intensa movilidad social queen la actualidad experimentan estas comunidades, ha disparado procesos dediferenciación interna y reducido el estatus del sistema decargos, al tiempo que ha incrementado la incidencia de conflictos intracomunitarios y puesto en crisis el antiguo concepto de comunidad. Más aún, en 
referencia al concepto tradicional de estado nación como una comunidad, Benedict A nderson (1991) afirma que aquellas nociones decamaradería horizontal comunitaria, solidaridad y homogeneidad cultural, son características que existen sólo en la mente delos miembros deesas comunidades, quienes en realidad viven en un medio pletórico de desigual dadesy explotación. Con esta consideración, Benedict concluyeque las comunidades románticamenteconstruidas en lamentedelosindividuos, más queser sociedades tangibles, son comunidades imaginadas.

En mi opinión, mucho antes de que tuvieran lugar entre los académicos estos cuestionamientos a las formas tradicionales de comunidad, la acción social de los yumanos había ya inaugurado formas de resistencia a ellas. Por esta razón, y considerando que la idea de comunidades sedentarias y sol idarias nunca ha existido entre estosgrupos, ni siquiera en la imaginación desus miembros, meparece queel concepto de comunidades inventadas describiría mejor el caso desuscomunidades, queel concepto deAnderson. Dehecho, tomando en cuenta las descripciones provistas por los misioneros españoles (Baegert, 1794; Venegas, 1739) y los pioneros en la etnografía de Baja California (Aschmann, 1959; Meigs, 1939), los yumanos precolombinos lejos devivir en estetipo decomunidades, seencontraban organizados en lo que Evans-Pritchard (1956) define como un sistema de linajes segmentarios. Esto es, en una serie de pequeños grupos dispersos, cazadores, recolectores, semi hostiles entre ellos, y con una multifragmentada autoridad patriarcal, que a pesar desus lazos de parentesco, cultural es y lingüísticos, observaban una el evada competencia por los escasos recursos del desierto. Incluso, debido a esta elevada competencia, los yumanos nunca desarrollaron un sistema de cargos ni un sistema cívico-ceremonial, y seencontraban migrando a lo largo de un territorio conocido ahora como el suroesteestadounidensey el noroeste mexicano, o más al lá de esta limitada área. Tal y como lo han comprobado las evidencias arqueológicas, esta intensa movilidad condujo a estos grupos a desarrollar un regular contacto con la cultura paquimé, los pima, pápago, eincluso con los dené de la región montañosa dela Columbia Británica en Canadá. ${ }^{2}$

Como es sabido, a pesar de los subsecuentes esfuerzos de los misioneros europeos y del gobierno mexicano por transformar a estos 
grupos en pueblos indios, los yumanos llegaron al final del siglo XIX manteniendo su movilidad. Después de quelas actividades misionales fueron clausuradas, David Golbaum (1984) inspeccionó el territorio indígena del norte deBaja California, y encontró quesólo unos cuantos linajes habían permanecido en los sitios misionales, viviendo en una seriedepequeños einestables caseríos. Deacuerdo con esteexplorador, debido a quela mayoría dela población nativa se había reagrupado en pequeñas bandas y retornado a su vida de tipo nómada, sólo habían quedado ruinas de las antiguas misiones: canales, cisternas y presas para irrigar la tierra, unas cuantas casas deadobe con techos deramas, eincluso cuevas, en donde sól o al rededor decien personassobrevivían practicando las actividades aprendidas de los misioneros y sus propias estrategias de supervivencia. Según Golbaum, estos indígenas practicaban una agricultura a pequeña escala, criaban animales y elaboraban objetos de piel, pero también, en su mayoría, cazaban animales y recolectaban plantas y miel silvestres, pescaban y manufacturaban sus tradicionales objetos decordelería, cestería y cerámica (Golbaum, 1984). Más aún, dado queel establecimiento de la nueva frontera en 1848 no afectó inmediatamente sus regulares desplazamientos, los yumanos arribaron al siglo xx como grupos observando una intensa movilidad, ahora transfronteriza. Tal y como lo expresa Delfina Cuero, en esta circunstancia, los indígenas pudieron seguir cruzando la línea fronterizasin restricciones durantelos primeros años, siendo usual quemiembros defamilias localizadas en ambos lados dela frontera se visitaran, asistieran a ceremonias, acudieran a recolectar bellotay piñón, eincluso, cambiaran su residencia en cualquier dirección. Posteriormente, con el incremento de la presencia de mineros y rancheros atraídos por la fiebre del oro en California, la movilidad de estos indígenas adquirió una dirección norte-sur, al encontrar en Baja California un "refugio natural" para continuar reproduciendo su tradicional forma de vida. Como lo afirma FlorenceShipeck con referencia específica a losk'miai,

${ }^{2}$ Al respecto, Meigs (1970) habla de las capas ceremoniales elaboradas con cabello humano, empleadas tanto por los kiliwa y cochimí deBaja California, y los denéde Canadá. 
...en el pasado ellos [los indígenas] se desplazaban libremente a lo largo del territorio K'miai para asistir a funerales, cere monias, e ir en las estaciones apropiadas a los eventos colectivos de recolección de alimentos. Ellos no sabían nada de la frontera internacional que disectaba su territorio justamente a la mitad. Ellos solamente sabían que la existencia indígena aún continuaba hacia el sur de su territorio (1968:11).

Años más tarde, sin embargo, debido a la expansión capitalista en Baja California, ese territorio dejó de ser tal refugio natural para los yumanos, y su movilidad adquirió una dirección sur-norte, al empezar a comerciar productos obtenidos de su actividad cazadora y recolectoraen el lado mexicano, con losyumanos reducidos en reservaciones indígenas en Estados Unidos (Hinton y Owen, 1957).

Sumado a la persistencia de su movilidad, y a pesar de los subsecuentes esfuerzos deagentes externos por imponer una autoridad central entre los yumanos, el sistema de linajes segmentario continuó existiendo. Como es deconocimiento común, los misioneros insistieron en construir una estructura deautoridad jerárquica y centralizada entre estos grupos indígenas, a través del nombramiento de gobernadores, mayores, capitanes y generales, sin embargo, los indígenas reaccionaron con cuestionamientos muchas veces mordaces a la imposición de esta estructura, como lo ilustra el siguientetestimonio del PadreGarcés de San Bernardino de los Opas:

También les propuse a los viejos juntasen la gente para que el señor comandante eligiese entre ellos, en nombre del rey, un gobernador y alcaldes, a lo que me respondió un viejo muy serio estas palabras: 'mira, el justicia es para castigar lo malo, y no siendo nosotros malos, ¿para qué es la justicia?' (Ochoa, 1976: 18).

Más aún, como lo explica Meigs (1939), aun cuando los yumanos Ilegaron a adoptar con el paso del tiempo esta estructura deautoridad, los indígenas rara vez le otorgaron poder al interior del grupo. Dado quela autoridad legitimada continuaba descansando en la cabeza de 
linaje, las autoridades nombradas necesariamente representaban específicamentea una deestas unidades, y por eso, su autoridad nunca pudo ser desarrollada como un poder central y legitimado por el resto de la población, menos aun por aquellos que pertenecían a linajes enemigos.

Final mente, la persistencia deeste sistema fragmentado duranteel periodo post-misional, l legó a ser evidenteen los eventos históricos de 1911, cuando las fuerzas revolucionarias invadieron Baja California. Como lo explica Owen (1963), al atravesar los magonistas el territorio yumano, los pai-pai de los linajes Kulwat, Wuatch y Xamwlch, y prácticamentetoda la tribu kiliwa seles unieron. Sin embargo, lost'pai deloslinajes Koa'l, M iakwas y Quashaqsh, y los kiliwa del linajeJepiPakawás, fueron reclutados por las tropas federal es para combatir en contra delos magonistas. Como esteautor lo ha destacado, la afiliación deestos linajes a uno u otro bando no obedecía a convicciones políticas propias, sino a una larga historia deotro tipo deconflictos entreellos. Owen argumenta que previo al arribo de los magonistas, estos dos grupos de linajes habían protagonizado una serie de desacuerdos debido a la ocurrencia de varias muertes, adjudicadas a la presencia deun chamán diabólico. Por esta razón, con el arribo de los invasores, estos grupos vieron la oportunidad de adquirir rifles y municiones, aliánd ose a al guna de las facciones en conflicto, y dar rienda suelta a sus tensiones.

No obstantela persistencia delaintensa movilidad yumana y desu organización segmentaria, la reforma agraria en M éxico impuso sobre estos grupos la noción de ejido, y por medio de esta acción las comunidades yumanas fueron oficialmente inventadas en 1935. Con el argumento de facilitar la aplicación de las políticas indigenistas y llevar a cabo una mejor distribución dela tierra y los beneficios sociales, el gobierno indujo a estos indígenas a definir su residencia en al guna de las comunidades indígenas creadas en Baja California. Desde entonces, aquellos quepermanecieron sin afiliación específica a alguno de estos asentamientos, empezaron a perder tangibilidad para efecto de las políticas indigenistas oficiales, que desde entonces dirigieron todos sus esfuerzos a fortal ecer la ya mencionada noción idealizada de comunidad, que incluía la supuesta organización colectiva de los 
pueblos agrícolas mesoamericanos. Así, en un intento por imprimir estas características a los asentamientos creados, el Instituto Nacional Indigenista (INI) en Baja California, empezó a destinar el noventa por ciento de sus recursos a la promoción de cooperativas agrícolas y ganaderas en estas comunidades (INI, 1986). A partir de entonces, los yumanos han sido vistos como indígenas comunitarios que viven asociados a alguno de estos ejidos sedentarios, observando una supuesta camaradería horizontal.

Sin embargo, la falta de éxito de las cooperativas, su patrón de asentamiento, la ausencia de una autoridad central, y sobre todo el éxodo masivo, contradicen la existencia real de dichas comunidades indígenas, en su concepción original. Al respecto, se debe reconocer, primero, queen estos ejidos la mayoría de los proyectos cooperativos han fracasado, y como evidencia deello podemos mencionar los casos delas cooperativas productoras demiel y jojoba, y la práctica agrícola. En el primer caso, se trata de un proyecto echado a andar a iniciativa del INI y el Programa Integral para el Desarrollo Rural en 1979, el cual tenía por objetivo desarrollar cooperativas productoras de miel, considerando que ésta era una de las actividades más antiguas desarrolladas por los yumanos. Como parte de este proyecto, a cada comunidad lefueotorgada infraestructura y apoyo técnico y financiero; sin embargo, de acuerdo con los técnicos del INI, la negligencia de los participantes en esta cooperativa a trabajar en equipo, resultó en la ausencia de una adecuada irrigación de los panales y finalmenteen su muerte. Desde entonces, la infraestructura de estas cooperativas se encuentra en ruinas y abandonada.

El segundo ejemplo se trata del proyecto de producción de jojoba en 1984. Tomando en cuenta la abundancia deestearbusto en lastierras indígenas, así como su elevada demanda en los Estados Unidos, el IN I intentó crear invernaderos de jojoba operados por cooperativas indígenas. Consecuentemente, la banca gubernamental otorgó apoyo financiero, y una moderna infraestructura fue establecida en cada comunidad para la domesticación de esta planta. Un equipo deagrónomos y administradores fue enviado de la ciudad de México para apoyar al programa, y se contrataron antropólogos para estimular y orientar a los yumanos a través de métodos audiovisuales, sobre como 
trabajar en cooperativas. Sin embargo, la escasa participación dela gente en este tipo de empresa, hizo imposi ble que este programa obtuviera Ios resultados deseados. Conflictos entre el equipo del INI y los indígenas aparecieron, la infraestructura permaneció en abandono, y partes del equipo y las reproductoras de video que habían sido traídas, por aquéllos que habían planeado románticamente el desarrollo de cooperativas en estas comunidades, fueron hurtadas (Domínguez, comunicación personal).

Finalmente, una tercer evidencia de estos constantes boicoteos a los proyectos cooperativos del INI, es el hecho dequeno más del $30 \%$ dela población nativa cultiva la tierra (Garduño, 1995). De acuerdo al INI (1986) esto se debe otra vez a la ausencia de cooperación entre las diferentes familias, la resistencia a trabajar en equipo, y a que las familias indígenas han utilizado para propósitos diferentes los recursos obtenidos de fuentes gubernamentales para el cultivo de la tierra y la crianza deanimales.

Un segundo factor que contradicela existencia decomunidades bajo su concepción tradicional entre los yumanos, es el patrón de asentamiento de sus ejidos. Quienes hayan visitado al guna vez una de estas comunidades, habrán notado que lejos de constituir pueblos compactos al interior de una determinada área geográfica, estos asentamientos poseen un patrón extremadamentedisperso, con caseríos separadosincluso por kilómetros.

En mi opinión, este patrón de asentamiento no es casual, sino que obedecea cierta lógica, vinculada a la persistencia del sistema delinajes segmentario. Por ejemplo, dentro del territorio kiliwa existen básicamente dos caseríos separados aproximadamente por 7 kilómetros; un caserío pertenecea la familia Espinoza, asociada al linajeJ apokel-k(a)wa (Hombres del Valle), mientras queel otro pertenecea la familiaUchurte, asociada al linajeChihuilinomitai (Hombres del Arroyo del Gato Grande). Como quedó en evidencia en un proyecto comunitario de 1975, los indígenas yumanos están conscientes del carácter segmentario de sus asentamientos. En dicho año, el INI, en conjunto con otros organismos gubernamentales, intentó echar a andar un programa de autoconstrucción devivienda en estas comunidades. Mientras queestos organismos otorgaban gratuitamenteel material necesario para la 
construcción, los indígenas debían proveer la fuerza de trabajo para construir sus propias casas. El plan gubernamental incluía, sin embargo, que las casas fueran construidas siguiendo un patrón de asentamiento compacto, con el fin defacilitar la introducción deserviciosala comunidad. La resistencia de los indígenas a participar en el programa fue evidente, y su argumento central puede resumirse en los siguientes testimonios:

Quévamos a hacer viviendo todos juntos? Nada; hay mucha tierra y muchos problemas entre nosotros (Cañedo, comunicación personal).

Cuando nos dieron las casas querían que viviéramos cerca uno del otro, pero no; muchos de nosotros tenemos esta costumbre; por lo menos yo soy feliz de vivir lejos de los demás, de esta manera se vive mejor. Viviendo todos apretados hay muchos pleitos (Vega, comunicación personal).

Por otra parte, el tercer factor que cuestiona la existencia real de comunidades yumanas es la ausencia de una autoridad central legitimada. Con la invención de los ejidos indígenas, se instituyó la figura del comisariado ejidal, reconocido oficialmentecomo la autoridad central querepresentaal conjunto dela comunidad en asuntos agrarios. Posteriormente, en los años setenta, bajo una orientación reformista del indigenismo, el gobierno mexicano instituyó el Ilamado Consejo Supremo delos Pueblos Indígenas, para lo cual promovió el nombramiento de jefes supremos que representaran a cada etnia en la organización nacional. Deesta manera, una nueva figura deautoridad (también con supuestas funciones centrales) fueintroducidaal interior de las comunidades yumanas. Desde entonces, en lugar de tener una sola supuesta autoridad central por comunidad, estos indígenas Ilegaron a tener hasta tres que competían por el liderazgo de los distintos grupos: Ios generales o capitanes introducidos por los misioneros, el comisariado ejidal introducido por la ReformaA graria, y el jefesupremo introducido durantelos años setenta. Dado quedichas 
autoridades podían llegar a pertenecer hasta a tres linajes diferentes, éstas nunca llegaban a ser vistas como agentes neutrales dentro de su comunidad; por el contrario, la suspicacia sobresus acciones empezóa ser algo común entrelos miembros delos demás linajes, quienes desde entonces empezaron a dar por sentado que estos líderes utilizan su posición para obtener el control de los recursos gubernamentales dirigidos hacia el ejido, para beneficiar a sus respectivas familias. Más aún, tal y como lo señalaba Owen en 1959, a pesar de que estas tres instancias de autoridad eran democráticamente electas, ninguna de ellas llegó a poseer el prestigio, el poder real y efectivo, quelas cabezas delinaje aún poseen en estas comunidades inventadas.

Finalmente, una indiscutible evidencia del fracaso de estas comunidades, essu incapacidad de retener a la mayoría dela población para la que fueron creadas. Ciertamente es difícil establecer un censo de aquella población indígena residente fuera de estos territorios ejidales, sin embargo, a través de mis actividades detrabajo decampo he podido constatar que por lo menos 750 personas pertenecientes a estos grupos y cuya edad oscila entre los 15 y los 50 años, vivefuera de ellos. Esto significa dos cosas, primero, que el mismo volumen de población residenteal interior deestosejidos, no seencuentra asociado permanentemente a al guna de las comunidades indígenas existentes, y dos, que en las comunidades inventadas residen princi pal mentelos más ancianos, es decir, las cabezas de linaje. Dealguna manera, estos dos hechos explican por qué, investigaciones previas han observado una población predominantemente anciana al interior de estas comunidades, concluyendo que en ellas se está dando una dramática caída en su tasa defecundidad, y por lo tanto un proceso de extinción indígena. Sin embargo, como se ha discutido hasta aquí, las comunidades yumanas en Baja California son sól o una ilusoria invención, y por lo tanto, es un error deducir que los indígenas yumanos se encuentran experimentando procesos de extinción y asimilación cultural a través de la disipación de dicha invención. Más aún, emplear esta invención para propósitos de investigación o para la toma de decisiones en la política indigenista, oscurecela existencia desegmentos dispersos de la población yumana que continúan manteniendo sus vínculos sociales y los elementos básicos de su organización social, 
lejos de estas comunidades. En la siguiente sección se argumenta el hecho de que los yumanos han reinterpretado el oscurecimiento (intencional o no) de su presencia, para desarrollar la construcción de comunidades queaparecen invisi bles antelos ojos delos antropólogos tradicionales y los agentes del gobierno, pero que son perfectamente tangibles para los mismos indígenas.

\section{LASCOMUNIDADESINVISIBLES}

Tradicionalmente, la persistencia de un grupo étnico ha sido asociada a la existencia de poblaciones geográficamente identificadas y con patrones cultural es decarácter distintivo (exóticos). Consecuentemente, la dispersión de estas poblaciones y la disipación de estos patrones supuestamente preexistentes, han sido relacionadas a la noción de asimilación cultural o mestizaje. En esta sección se sostiene, sin embargo, que los yumanos de Baja California son invisibles ante esta obsoleta y tradicional perspectiva etnográfica, pero étnicamente persistentes si empleamos las nociones de hiper espacio y auto diferenciación étnica: así, desde una perspectiva interna, los yumanos constituyen comunidades invisibles quecontradicen las construcciones hegemónicas impuestas.

Durante los años sesenta y setenta, la idea de comunidades autoconteni das condujo a los antropólogos a establecer un "inevitable" vínculo entre dispersión, integración económica a la economía capital ista, y asimilación cultural. Desde esta perspectiva, sellegó a la conclusión dequeaquel los grupos con preexistentes economías basadas en actividades dispersas e individuales (como las delos yumanos), se encontraban más prodives a experimentar tal asimilación, queaquellos con preexistentes economías basadas en actividades colectivas. Ejemplo deesta perspectiva es el estudio comparativo realizado por Murphy y Stewart, sobre los mundurucu del Brasil y los al gonkianos del noreste deCanadá; en este estudio lo autores concluían que en una situación decontacto cultural eintegración capital ista, la estructura de la cultura nativa pertenecientea una sociedad no estratificada, y cuyos miembros sobreviven de la recolección individual de productos silvestres 
encontrados en una extensa distribución, tiene más posibilidades de ser destruida (M urphy y Stewart, 1977:178).

Al final delos años ochenta, la noción de comunidades geográfica y culturalmentedelimitadas fueseriamentecuestionada, pero finalmente, el análisis social continuó estando centrado en unidades de población geográficamentelocal izadas y distinguibles. Tal y como lo señalan los teóricos del transnacionalismo (Gupta y Ferguson, 1992; Richmond, 1988; Georges, 1990), la intensificación de la migración transnacional ha conducido a los antropólogos a poner más atención en la construcción de redes sociales como un método clave para evaluar el persistente sentido de comunidad entre aquellas poblaciones dispersas a lo largo de dos o más naciones. Sin embargo, tal y como lo reconoce Kearney (1996), en estas nuevas el aboraciones, las redes sociales fueron asumidas como mecanismos de interacción cara a cara en lugares específicosy como medios para aumentar la dimensión de los espacios, a través de los cuales la gente migra. De esta manera, si durante las décadas de 1970 y 1980 se hablaba de comunidades expulsoras y receptoras (Stahl, 1988; Cornelius, 1987-1990; Bustamante, 1989), durantelos años noventa los nuevos lugares específicos llegaron a ser las comunidades vástago, resultado dela tendencia a la concentración, o a la construcción de barrios de migrantes en las zonas de destino. Para Leo Chávez (1990), estas comunidades vástago pueden ser definidas como entidades territorial mentelocal izadas, establecidas por amplias redes social es entre familiares y amigos, así como por nuevas relaciones creadas en el nuevo lugar de asentamiento, con el fin de proveer al migrante de apoyo estratégico para su desenvolvimiento. Así, dado que estas comunidades vástago implican la existencia de al tas concentraciones de gente, su localización se da principalmente en áreas con una elevada demanda de fuerza de trabajo. Más aún, dado que los habitantes de estas comunidades vástago se encuentran asociados por leal tades étnicas, éstas constituyen compactos asentamientos culturalmentedistinguibles.

Recientemente, Kearney (1996) ha sugerido sin embargo, que la actual experiencia migratoria secaracteriza por la construcción dehiper espacios difícilmentevisibles. Deacuerdo con esteautor, una red social debe entendersehoy día como un componente de la comunicación en 
la cual el elemento humano puede ser potencializado por avances electrónicos, que disuelven las fronteras espaciales. Desde esta perspectiva, una red social no solamente facilita la migración de seres humanos sino también de signos y otros valores que se desplazan a través de un espacio sin polos y centros territorial mentetangibles. De esta manera, Kearney argumenta que la morfología de una red social constituida en hiper espacio es la deuna amiba, es decir, la deuna criatura con una compleja diferenciación interna, pero sin células visibles ni órganosquecorrespondan alos componentessociales deuna comunidad corporada; de esta manera también, un hiper espacio, como la amiba, puede extenderseen cualquier y hacia toda dirección (1996:125).

Desdemi punto devista, esto es exactamentelo queestá sucediendo con los grupos yumanos: La construcción de un hiper espacio a través del cual simultáneamente reproducen y trascienden a su sistema segmentario, con la finalidad de generar comunidades invisibles. Debido a la persistencia desu sistema delinajes, losindígenasyumanos queviven fuera delos territorios ejidales no tienden a integrar visibles asentamientos compactos o comunidades vástago, como lo hace la mayoría de los grupos migrantes estudiados hasta ahora. En primer Iugar, la evidencia obtenida de poblaciones con antecedentes de economías cazadoras y recolectoras, nosindica quesu tasa denatalidad se reduce a un descendiente cada tres o cuatro años (Symons, 1979). Por esta razón, el tamaño de las poblaciones yumanas nunca será semejanteal tamaño delas poblaciones de aquellos grupos indígenas de tradición sedentaria y agrícola como los mixtecos o zapotecos. En segundo lugar, la movilidad delosyumanos continúa estando limitada a pequeños grupos de apenas tres o cuatro personas, quesemantienen observando una movilidad constantea lo largo de veinticinco destinos diferentes, que no necesariamente poseen una elevada demanda de fuerza detrabajo. Dehecho, lejos demigrar hacia las áreas industriales y urbanas, como Tijuana o Chicago, o a los lugares con una intensa actividad agrícola, como San Quintín o a los valles agrícolas de California, los yumanos se desplazan básicamentea lo largo de pequeños ranchos ganaderos, dispersos en las montañas dela Sierradejuárez - San Pedro Mártir, y las reservaciones indígenas localizadas en las inmediaciones de Campo, Viejas, Cuyamaca, etcétera, en California. 
Por esta razón, a diferencia de lo que ocurre con los grupos sedentariosy agrícolas, los yumanos queviven al exterior delos ejidos indígenas tienden a permanecer dispersos einvisibles.

Sin embargo, pesea su invisibilidad, es evidentela persistencia dela unidad básica dela organización social yumanafuera delascomunidades indígenas. Tal y como lo ha demostrado Florence Shipeck (1968), dado que los nombres de los linajes indígenas fueron transformados en apellidos con una cercana pronunciación en español, aún es posible identificar que los Kwal son los Cuero, los Ae,sun son los Osuna, los X.tam son los Tambo, etcétera. Por otra parte, es también evidente que estos linajes, con presencia fuera y dentro de los ejidos indígenas, se encuentran vinculados a través de relaciones de parentesco y amistad, constituyendo canales de información que les permiten actualizar permanentementesu catálogo deoportunidades deempleo, deasistencia médica gratuita, o tener conocimiento acerca dela cel ebración deeventos tales como rodeoso los periódicosy ahora transnacionales Pow-Wowso Kuri-Kuris, que son organizados ya sea en California, Arizona, o Baja California. Deesta manera, aunquela organización básica delosyumanos es el sistema segmentario, estos indígenas se encuentran actualmente comprometidos en la construcción de comunidades indígenas transnacionales queson socialmenteilimitadasy dispersas, a través deespacios sin ejes reconocibles, o como diría Kearney, a través deun hiper espacio queno se encuentra anclado en un sitio específico.

Pero, de que tipo de invisibilidad estamos hablando aquí. Para precisar este concepto, echemos un vistazo a las elaboraciones más tempranas al respecto. En los años sesenta, Abner Cohen (1969) desarrolló la noción de organizaciones invisibles, la cual sin embargo, relacionó de manera poco afortunada al concepto de distintividad cultural y a la noción de restrictividad ocupacional. A través de un análisis comparativo sobrel os procesos experimentados por los hausa y los ibo, al migrar hacia Ibadán, Nigeria, este autor concluyó que el primero de los grupos había sido capaz de construir organizaciones invisibles, al adaptar su estructura tradicional a los requerimientos organizacional es de una actividad económica específica: el comercio a larga distancia, entre la sabana y la región boscosa. Por el contrario, Cohen observó quelosi bos habían empezado a experimentar laerosión 
de su organización tradicional, inducida por un proceso de diferenciación ocupacional, al contratarse como mecánicos, dependientes, trabajadores de imprenta, profesoresy empleados detodo tipo. Deesta manera, este autor concluye que mientras que los hausa habían sido capaces de preservar su distintividad y profundizar su identidad y "exclusividad cultural", a través de ajustar sus vidas a las actividades de un particular nicho económico, la distintividad y la identificación étnicas del os ibos había si do severamente debilitada, o hechas innecesarias ante su nueva situación ocupacional y de clase; lo cual vino a disolver definitivamentelas fronteras étnico-culturales deesteúltimo grupo. Así, el tipo de invisibilidad discutida por Cohen, en el caso de las organizaciones étnicas de los hausa, resultó ser no del todo invisible, al relacionar la persistencia étnica con la noción dedistintividad cultural.

Una noción diferente de invisibilidad, elaborada en reacción a la anterior perspectiva transcultural, es la sugerida por Kearney en los años ochenta, al hablar de "la mano invisible y el hecho visible". En referencia a la existencia deredes articuladoras demigrantes, esteautor contradijo la supuesta relación que previamente se había establecido entre restrictividad ocupacional, persistencia dela estructura degrupo, y etnicidad. Deacuerdo con Kearney, estas redes conducen a los individuos hacia fuera de sus comunidades de origen, al tiempo que les permiten desplazarsey mantener los nexos a lo largo de diversos tipos de espacios, rurales, urbanos o suburbanos, en donde los migrantes experimentan una completa o parcial proletarización, o sobreviven ocupándose en diversas actividades que incluyen los sectores informales de la economía. Diez años más tarde (1996), Kearney consolidó esta idea con su noción del polibio, el cual, en contraste a los organismos que se han adaptado a vivir en un solo medio físico, o a los anfibios, que sobreviven en dos medios diferentes, constituye un tipo de migranteque ha llegado a adaptarse a una gran diversidad demedios sociales, reestructurando permanentementesu etnicidad, en relación al contexto socio-cultural en que se encuentre. Así, el polibio transciende las dualidades tradicionales de urbano-rural, nacionalinternacional, proletario-campesino, o indígena-no indígena, y subvierte la noción de identidades monolíticas, estáticas y visibles (Kearney, 1996: 142). 
En mi opinión los indios yumanos de Baja California constituyen un buen ejemplo delo queson los polibios, ya quelejos deconcentrarse trabajando sólo en un nicho económico, tienden a diversificar sus ocupaciones, adaptando su estructura y etnicidad a esta dinámica. De la muestra estudiada en anteriores investigaciones, el $44 \%$ se encuentran ciertamente trabajando como vaqueros, pero el $20 \%$ desempeña tareas como trabajadores agrícolas, otro $20 \%$ como empacadores de atún, un $4.5 \%$ como ladrilleros, y un $2.5 \%$ como pescadores. Así, en lugar de ocupar un mismo segmento ocupacional, los yumanos se ocupan en diferentes posiciones dentro dela nueva estratificación social, y a pesar de experimentar esta diferenciación social, como ha sido explicado anteriormente, su sistema segmentario ha persistido. Por otra parte, dado su permanentetranscurrir a lo largo de diversos escenarios étnico-culturales, y consecuentemente a su constante relación subordinada con el Otro, la etnicidad delos grupos yumanos, en lugar dedesaparecer, persiste de una manera deterritorializada. Esto es, que independientemente de donde se encuentren trabajando o viviendo, estos indígenas continúan identificándose entre ellos como pai-país, kiliwas, cucapas, k'miai, o cochimís. A demás, una manera deestablecer formas de asociación adecuadas para manipular su situación de subordinación, es la creación y manipulación de múltiples identidades. Como hemos podido percatarnos, tan pronto setorna necesaria la estrategia de reagrupar grandes colectividades, el recurso de autoreferencia entre los yumanos deja de ser el origen étnico para convertirse en el origen geográfico, identificándose eventualmente como serreños, en el caso de aquellos que viven en la sierra, y rieños en el caso deaquellosqueviven en las inmediaciones del Río Colorado. ${ }^{3}$ Pero, más allá de la restrictiva identidad proveniente del sistema de

${ }^{3}$ Lorena Pérez (1990) nos presenta un ejemplo muy ilustrativo de este fenómeno entre los mazahua que viven en Ciudad Juárez, quienes se identifican a sí mismos ante los locales como tarahumaras (la etnia del estado), con el fin de evitar una extraordinaria discriminación y su posible deportación al Estado de México. 
linajes, los yumanos han desarrollado una nueva identidad que ha adoptado a las comunidades inventadas como su principal recurso; en este caso, a pesar de que la mayor parte de la vida de una persona haya transcurrido fuera de su comunidad de referencia, ésta puede llegar a identificarse a sí mismo como catarineño, si sus ancestros son de Santa Catarina, huerteño si son de La Huerta, zorreños si son de San José dela Zorra, etcétera. Por último, tal y como Glick, et al. (1992) y Varese (1994) lo han descrito en el caso deotros grupos migrantes, el transcurrir subordinado de los yumanos a lo largo de distintos escenarios, ha incrementado sus semejanzas entre sí, y reducido sus diferencias lingüísticas y culturales. Por esta razón, sobre las fragmentadas y parroquiales autodiferenciaciones múltiples, actualmente se desarrolla entre estos indígenas una identidad panétnica y extranacional, que consiste en el hecho de que, independientementedel origen étnico o geográfico, o de la residencia de la cabeza de linaje, estos grupos se identifican a sí mismos como "nosotros, los paisanos" versus "ustedes, los mexicanos o los gringos".

Estos hechos me permiten concluir lo siguiente. Ciertamente, los indígenas yumanos provienen deun preexistentetipo de sociedad no estratificada, con una economía sustentada en actividades individuales y dispersas, según la descripción de M urphy y Stewart. Ciertamente también, los yumanos se encuentran atravesando por un proceso de dispersión, proletarización y diferenciación ocupacional, como lo describen estos mismos autores y A bner Cohen. Seguramente, si aplicamos la noción de distintividad cultural para evaluar la persistencia étnica delos yumanos, en el contexto deestos fenómenos, concluiremos en anunciar la virtual desaparición de sus fronteras étnico-culturales; pero si atendemos a la reelaboración desu sistema segmentario a través dela definición de un hiper espacio, como a la deconstruccion de una múltiple auto referencia étnica, nos percataremos no sólo de la tangibilidad dedichasfronteras, sino dela mani pulación delas mismas, configurando así, comuni dades invisibles sobrela basededicho sistema segmentario. Por eso, mepermitiría afirmar queestos grupos indígenas no se encuentran experimentando un proceso de asimilación cultural en los términos tradicionalmente entendidos, sino: primero, una histórica resistenciaa la imposición dela noción convencional dePueblo 
Indio y comunidad; segundo, la subversión dela noción antropológica tradicional de distintividad cultural, a través del desarrollo de una intensa movilidad multidireccional y nuevasformas deauto referencia; y tercero, una resistencia a las tendencias hegemónicas quesegregan a los yumanos tanto en México como en los Estados Unidos, por medio deladeconstrucción demúltiplesidentidades deterritorializadasy panétnicas. Por supuesto, todos estos factores hacen quelos yumanos sean invisibles ante los ojos del Estado y los antropólogos tradicionales.

\section{CONCLUSIONES}

Entre los antropólogos quetrabajan el norte deM éxico sediceque "la antropología en M éxico está meso americanizada". En mi opinión, esta afirmación no es solamente válida como crítica a la antigua antropología mexicana, sino también a la moderna antropología, la cual paradójicamentesugierela trascendencia de los espacios geográficos, al tiempo querestringesu estudio a los grupos con patrones migratorios muy similares. Como ha sido mencionado al principio de este trabajo, las recientes elaboraciones de la antropología transnacional se han sustentado en el estudio de grupos procedentes de Meso A mérica, el Caribeo África, ignorando a quienes han sido los protagonistastempranos delos procesos transnacionales: los indígenas fronterizos. Sin embargo, estoy convencido que una reorientación de la nueva antropología hacia el estudio de los indígenas fronterizos, puedearrojar nuevas revelaciones sobre los procesos migratorios. Con respecto al caso específico de los yumanos de Baja Cal ifornia, una deestas revelaciones puedeser la refutación a los conceptos deextinción indígena o asimilación cultural, como fenómenos tradicionalmente adjudicados al conjunto delos indios peninsulares. Otra revelación podría ser el descubrimiento de los yumanos como grupos pioneros en la resistencia a la imposición dela noción convencional decomunidad o pueblo indio, incluso, mucho antes de que tuvieran lugar los cuestionamientos hechos por los académicos a la noción de comunidades delimitadas y autocontenidas. Como fue explicado en estetrabajo, una delas formas de esta resistencia podría ser la deconstruccion de la invención gubernamental del ejido, a través desu transformación en residencia decabezas de 
linaje; otra forma consistiría en la reelaboración de su sistema segmentario y etnicidad, para construir sobre ambos, un tipo de comunidad queescapa a la visibilidad del estado y los antropólogos clásicos. Estas comunidades, que en este trabajo han sido denominadas invisibles, están constituidas por una población detamaño reducido, queademás seencuentra permanentementemigrando demanerafragmentada en pequeños grupos, a lo largo deuna seriedepequeñas áreas rurales con una escasa demanda de fuerza de trabajo, y desarrollando una práctica social que aún está por ser documentada. De esta manera, mientras queel INI continúa tratando detransformar a los yumanos en comunidades agrícolas y cooperativas, bajo la noción nacionalista y post-revolucionaria de ejido, estos grupos han construido un tipo de comunidad que seextiende por encima dela frontera M éxico-Estados Unidos, subvirtiendo la noción transcultural de distintividad cultural, como el indicador claveparaidentificar la persistencia deun grupo étnico.

Finalmente, a manera de epílogo, me permito sugerir la reinterpretación del popular relato sobre Jal kutat, el terrible monstruo con una larga lengua defuego, como icono delos procesos aquí discutidos. Tal y como lo relataba Benito Peralta (q.p.d.), Jalkutat vivía en las inmediaciones de un pequeño arroyo localizado en Santa Catarina, amenazando con el extermi nio a toda persona y animal queseacercase a beber agua. Un día, un indio proveniente del sur, seriamente hirió a Jal kutat, quien trató de atrapar al indio con su enormelengua defuego; pero éste, hábilmente se despojó de sus sandalias, de su arco y de sus flechas, desu tocado y del resto desu indumentaria, y para confundirlo, fuearrojando estos artefactos, uno a uno, a la lengua de fuego de aquel monstruo. Jalkutat devoró las pertenencias del indio, y posteriormente sefueo murió, creyendo queen realidad había atrapado a su victimario. Sin embargo, desplazándose rápidamente, ya sin su vestimenta tradicional, este hombre pudo sobrevivir por el resto de sus días. ${ }^{4}$

${ }^{4}$ Síntesis no literal dela versión relatada por Benito Peralta, comunicación personal. 
Anderson, Benedict (1991). Imagined Communities. Nueva York: Verso. Aschmann, Homer (1959). "The central desert of Baja California", D emography and Ecology. University of California Press. Berkeley. A ppadurai, A rjun (1991). "Global ethnoscapes: N otes and queries for atransnational anthropology". Recapturing A nthropology. W orking in theP resent. R. Fox, ed. School of A merican Research Press. Santa Fe. Pp. 191-210.

Baegert, Juan Jacobo (1942). N oticias de la Península A mericana de California, 1794. Porrúa. México, D.F.

Basch, Linda, Glick Schiller and Szanton Blanc (1994). N ations U nbound:

Transnational Projects, Postcol onial Predicaments, and D eterritorialized

$\mathrm{N}$ ation-States. Gordon and Breach Publishers. Langhorne.

Bustamante, Jorge (1989). "Frontera M éxico-Estados Unidos:

Reflexiones para un marco teórico". Frontera N orte 1: 7-24.

Cancian, Frank (1992). The Decline of Community in Zinacantan. University Press. Sanford, CA.

Chambers, Ian (1994). M igrancy, culture, identity. Routledge. Nueva York.

Chávez, Leo R. (1990). “Coresidence and resistance: Strategies for survival among the undocumented Mexicans and Central Americans in theUnited States". U rban A nthropology 19(1-2): 31-61. (1992). "Shadowed Lives: Undocumented Immigrants". A merican Society. Harcourt Brace College Publishers. California.

Cohen, A bner (1969). Custom and Politics in U rban A frica: a Study of H ausa M igrants in Y oruba Towns. Rouledge and K. Paul. London.

Cook, S.F. (1937). "The extent and significance of disease among the Indians of Baja California 1697-1773". I bero-A merican 12 (1). University of California Press. Berkeley.

Cornelius, Wayne (1987-1990). "Labor migration to the United States: Development, outcomes, and alternatives in Mexican sending communities". U nauthorized M igration: A ddressing the Root Causes: Research Addendum, Commission for the Study of International Migration and CooperativeEconomic Development. Washington, DC. 1: 81-124. 
Dieguet, Leon (1978). “Etnografía deBaja California”. Cal afia 3 (5).

Evans-Pritchard, E.E. (1956). "The Nuer of Southern Sudan". A frican

Political Systems. Fortes Meyer and E. E. Evans, eds. Oxford:

London Pub., Oxford University Press. Pp. 272-296.

Garcia, Canclini Nestor (1989). Culturas híbridas. Estrategia para entrar y salir dela modernidad. Conaculta y Editorial Grijal bo. México, D.F. Garduño, Everardo (1995). En donde se mete el sol. H istoria y situación actual de los indígenas montañeses de Baja California. México, D.F.: Conaculta.

Georges, Eugenia (1990). The M aking of a Transnational Community: M igration, Development, and Cultural Change in the Dominican Republic. Columbia University Press. New York.

Glick, Nina, Linda Basch and Cristina Blanc-Szanton (1992). "Towards a transnational perspectiveon migration". Race, Class, Ethnicity, and $\mathrm{N}$ ationalism Reconsidered. TheN ew York Academy of Sciences. New York.

Golbaum, David (1984). “N oticia respecto alas comunidadesindígenas que pueblan el Distrito Nortedela Baja Cal ifornia." Calafia V (3): 3-5.

Gupta, A khil y James Ferguson (1992). “Beyond culture: Space, identity, and the politics of difference". Cultural A nthropology 7 (1): 6-23.

Hinton, Thomas y Roger Owen. 1957. "Some surviving groups in northern Baja Cal ifornia, Mexico". A merica Indígena 17 (1).

INAH (Instituto Nacional deA ntropología eHistoria) (1972). “Censo de los Grupos Indígenas de Baja Cal ifornia". Departamento de Etnología y A ntropología Social. Mexicali, mimeo.

_ 1978. "Censo de los Grupos Indígenas de Baja California". Departamento de Etnología y Antropología Social. Mexicali, mimeo.

INI (Instituto Nacional Indigenista) (1982). "Las Comunidades Indígenas deBaja Cal ifornia". Centro Coordinador Indigenista. Ensenada, mimeo.

- (1986). “Estrategia para Trabajo con los Grupos Indígenas de Baja California". Centro Coordinador Indigenista. Ensenada, mimeo. 
(1991). “Censo delas Comunidades Indígenas deBaja California”.

Centro Coordinador Indigenista. Ensenada, mimeo.

Kearney, Michael (1986). "From the invisible hand to visible fact: A nthropol ogical studies of migration and development". A nnual Review of A nthropology 15: 331-361.

- (1991). "Borders and boundaries of state and self at the end of empire". Journal of H istorical Sociology 4 (1): 52-74.

(1995). "The local and the global: The anthropology of globalization and transnational ism". A nnual Review of Anthropology 24: 547-65.

(1996). Reconceptualizing the Peasantry. Anthropology in Global Perspective. Westview Press. Boulder.

Laylander, Don (1991). “Organización comunitaria de los yumanos occidentales: una revisión etnográfica y prospecto arqueológico". Estudios F ronterizos 24-25.

León-Portilla, M iguel (1983). “Los primeros californios: Pre-historia y etnohistoria". Panorama H istórico de Baja California. Mexicali: UNAM/ UABC.

_ (1985) "Los indígenas de la frontera entre México y los Estados Unidos", Reglas del juego y juego sin reglas en la vida fronteriza. III Reunión de Universidades de México y Estados Unidos. Eds. Mario Miranda y James Wilkie, A nuies/ Profmex, México, D.F. Martínez, Pablo (1991). H istoria de Baja California. La Paz: Consejo Editorial del Gobierno del Estado de Baja Cal ifornia Sur.

Martínez, Zepeda Jorge (1985). “Música indígena en Baja California”. Mimeo.

Massey, William C. (1949). "Tribes and languages of Baja California”. Southwestern Journal of Anthropology 5 (3): 272-307.

Meigs, Peveril (1939). "The Kiliwa indians of lower California". I beroA merican 15. Berkel ey: University of California Press.

(1970). "Capes of human hair from Baja Cal ifornia and outside". Pacific Coast A rchaeol ogical Society Quaterly 6 (1): 21-28.

Murphy, RobertF. y Julian H. Steward (1977). “Tappers and trappers:

Parallel processes in acculturation". En Evolution and Ecology, Julian H. Steward, ed. Urbana: University of Illinois Press. Pp. 151-79. 
Ochoa, Jesús A. (1976). “Caciques, señores, capitanes y gobernadores. Nombramientos indígenas en Baja California". Calafia III (3). Universiad Autónoma deBaja California.

(1979). “Distribución actual de los grupos indígenas de Baja California". Calafia IV (1).

Owen, Roger (1959). "The Indians of Santa Catarina, Baja California Norte, Mexico: Concepts of Disease and Curing". Ph.D. Dissertation. Los Angeles: University of California.

— (1963). "Indians and revolution: the 1911 invasion of Baja California, M exico". Ethnohistory 10 (4).

Pérez, Maya Lorena (1990). "Ser mazahua en Ciudad Juárez". M éxico Indígena 4: 21-25.

Richmond, A nthony H. (1988). Socio-Cultural A daptation and Conflict in Immigrant-R eceiving Countries in International M igration Today. Charles Stahl, ed. Pp. 109-124. Belgium: Unesco, University of Western A ustralia, Centrefor Migration and Development Studies.

Rouse, Roger (1989). M exican M igration to the United States: Family Relations in the D evel opment of a Transnational M igrant Circuit. Diss. Stanford University.

_ (1991). "Mexican migration and the social space of postmodernism". Diaspora 1 (1): 9-23.

(1992). "Making sense of settlement: Class transformation, cultural struggle, and transnational ism among M exican migrants in the United States in towards a transnational perspective on migration. Race, Class, Ethnicity, and Nationalism Reconsidered". N. Glick Schiller, L. Basch, and C. Blanc-Szanton, eds., New York Academy of Sciences. Nueva York. Pp. 25-52.

Sassen, Saskia (1998). Globalization and its discontents. The New Press. Nueva York.

Seton-Watson, Hugh (1977). N ations and States. Westview Press. Boulder. Shipek, Florence (1968). TheA utobiography of D el phina Cuero, a D iegueno Indian. LosA ngeles: Dawson's Book Shop, Baja California Travel Series 16,

Stahl, Charles W. (1988). International M igration Today. Charles Stahl ed., Belgium: Unseco, University of Western Australia, Centre for Migration and Development Studies. 
Symons, Donald (1979). The Evolution of Human Sexuality. Oxford: Oxford University Press.

Varese, Stefano (1994). “Entreel tianguis y los designios neo-imperiales: Etnopolítica de la migración transnacional indígena”. Coloquio La Ruta Mixteca. A gosto 2-3. Mimeo.

Velez-Ibanez, Carlos G. (1996). Border V isions. M exican Cultures of the Southwest U nited States. Tucson: The University of A rizona Press. Venegas, Miguel (1943). N oticia de la California y de su conquista temporal y espiritual, 1739. Editorial Layac. México, D.F.

Wolf, Eric (1957). "Closed corporatecommunities in M esoamerica and Java". Southwestern Journal of A nthropology 13(1): 1-18.

\section{COMUNICACIÓN PERSONAL}

Cabello, Carlos; Técnico agrónomo (INI)

Cañedo, Carlos; Informante pai-pai

Carbajal, Normal; Trabajadora social (INI)

Cortez, Edna; Bióloga

Dominguez, Anselmo; Informante t'pai

Peralta, Benito; Informante pai-pai

Uchurte, Trini; Informante kiliwa

Vega, Andrés; Informante k'miai

Zárate, David; Historiador 